OPEN ACCESS

Edited by:

Silvia Testa,

University of Aosta Valley, Italy

Reviewed by:

Dominic Willmott,

Manchester Metropolitan University,

United Kingdom

Giorgia Molinengo,

University of Turin, Italy

${ }^{*}$ Correspondence:

Marcello Passarelli

marcello.passarelli@itd.cnr.it; marcello.passarelli@gmail.com

Specialty section: This article was submitted to

Quantitative Psychology and Measurement, a section of the journal

Frontiers in Psychology

Received: 20 March 2020

Accepted: 19 June 2020

Published: 23 July 2020

Citation:

Casetta L, Rizzi L, Passarelli M, Arcara G and Perrella R (2020) Italian Validation of the Touch Avoidance Measure and the Touch Avoidance

Questionnaire.

Front. Psychol. 11:1673. doi: 10.3389/fpsyg.2020.01673

\section{Italian Validation of the Touch Avoidance Measure and the Touch Avoidance Questionnaire}

\author{
Laura Casetta ${ }^{1}$, Luca Rizzi ${ }^{1}$, Marcello Passarelli2*, Giorgio Arcara ${ }^{3}$ and \\ Raffaella Perrella ${ }^{4}$
}

${ }^{1}$ Associazione Centro di Psicologia e Psicoterapia Funzionale, Istitituto S.I.F., Padua, Italy, ${ }^{2}$ Institute of Educational Technology, National Research Council, Genoa, Italy, ${ }^{3}$ IRCSS San Camillo Hospital, Venice, Italy, ${ }^{4}$ Department of Psychology, University of Campania "Luigi Vanvitelli," Caserta, Italy

Social touch is essential in relationships and well-being, but the unique personal experience of touch is not assessed and taken into account in health and social care services. The pleasantness of gentle stroking is influenced by gender, toucher genre, toucher familiarity, culture, and age. Moreover, pleasantness is influenced by touch avoidance, the attitude toward interpersonal touch. The aim of this article is to present the translation, adaptation, and validation in Italian of two scales to measure touch avoidance. For translation and validation, we selected the most used scale, the Touch Avoidance Measure (TAM) and a more recent scale, the Touch Avoidance Questionnaire (TAQ). Confirmatory factor analyses reported good model fit for the TAM [comparative fit index $(\mathrm{CFI})=0.947$, Tucker-Lewis index $(T L I)=0.940$, root-mean-square error of approximation $(\mathrm{RMSEA})=0.065]$ and excellent model fit for the $\mathrm{TAQ}(\mathrm{CFI}=0.954$, $\mathrm{TLI}=0.950, \mathrm{RMSEA}=0.058$ ). Internal consistency was high for all subscales, except the TAQ "Stranger" subscale. One-month test-retest reliability ranged from 0.67 to 0.90 for each subscale. Lastly, convergent validity between the TAM and TAQ was also found to be high. We conclude that the TAM and TAQ can be used to assess touch avoidance with Italian samples. The instrument can be used to support healthcare professionals and to assess attitudes toward touch in individuals with interpersonal difficulties.

Keywords: touch avoidance, social touch, ordinal CFA, non-verbal communication, Italian validation

\section{INTRODUCTION}

Touch is one of the most important senses for survival: it is one of the first to develop in the maternal womb and the most developed at birth (Hertenstein et al., 2006). Touch has several functions: it helps to discriminate the location of a stimulus on the skin surface, to examine objects haptically, and to manipulate them. Touch also serves the function to shape an integrated sense of our body (Serino and Haggard, 2010). Moreover, touch has a communicative function, as distinct emotions can be communicated and correctly decoded through touch (Hertenstein et al., 2006, 2009).

In addition to this, touch in the two last decades has been recognized for its important social function, to the point of calling the skin a "social organ" (Morrison et al., 2010). This highlights the importance of one characteristic of the sense of touch: its pleasantness. Pleasantness of touch has been explained through the social touch hypothesis (Olausson et al., 2010) a theory that explains that slow gentle stroking is pleasant because it is important in close affiliative interactions. 
The pleasantness of touch is due to the C-tactil (CT) afferents pathway, an unmyelinated, slow conducting (0.6$1.3 \mathrm{~m} / \mathrm{s}$ ), low-threshold mechanoreceptor that only innervates hairy skin (Vallbo et al., 1999; McGlone et al., 2014). In addition to the neurophysiological basis of pleasantness connected to gentle stroking, personal and contextual factors influence the response of pleasantness. For example, receiving a gentle stroke by an undesirable toucher changes the pleasant experience to disgust (Ellingsen et al., 2016). Furthermore, when blindfolded heterosexual men were caressed by a woman, they responded with pleasure, while if they were told they were being caressed by a man (even if the same woman was stroking them), they responded with aversion (Gazzola et al., 2012; Scheele et al., 2014).

Culture also has an important influence regarding attitudes toward touch. For example, in Italy, it is common practice to greet someone with a kiss on both their cheeks or with a hug. On the contrary, in Japan, bowing is the traditional, customary greeting; there is no physical contact between individuals (McDaniel and Andersen, 1998; Finnegan, 2005). Dibiase and Gunnoe (2004) examined both hand and non-hand touches in Italy, the Czechia, and the United States. Results show that Czech men touch more than any other group, while Czech and Italian women and Italian men use non-hand touch more than the other groups. Authors suggest that these results are partially explained by dominance theory, claiming that touching behavior is an expression of dominance. Dominance is associated not only with social status but also with gender, especially in countries where men are still in clearly dominant positions (Dibiase and Gunnoe, 2004).

When considering personal factors, chronic pain, depression, and anhedonia can reduce the hedonic experiences connected to affiliative touch (Pizzagalli et al., 2008; Elvemo et al., 2015; Thomsen, 2015). Even anxiety can reduce pleasantness connected to touch. Wilhelm et al. (2001) demonstrated that high anxiety women, as compared to low anxiety women, following a 2-min touch on the wrist by a male experimenter, reported greater anxiety and embarrassment.

Age is another factor influencing the pleasantness of touch: affective touch is perceived as more pleasant at a young age (Sehlstedt et al., 2016; Croy et al., 2019). Even gender influences attitudes toward touch: in general, women respond more positively than men to touch (Ozolins and Sandberg, 2009). Furthermore, women experience more pleasantness when touched by strangers in a non-sexual way (Hall et al., 2005), while men prefer being touched by women rather than by men (Gazzola et al., 2012; Scheele et al., 2014). Additionally, men avoid touch significantly more than women toward partner, family, and same-sex individuals (Ozolins and Sandberg, 2009).

Lastly, people vary in their base predisposition toward being touched. The touch avoidance construct is an index of a person's attitude toward touching and being touched (Andersen and Leibowitz, 1978). Touch avoidance reduces the perceived pleasantness of all kinds of touch (Hielscher and Mahar, 2017). This predisposition develops throughout a person's lifetime and is a stable personality trait (Johansson, 2013). Cultural and biological factors interacting with early experiences with touch within the family influence attitudes toward touch. According to attachment theory, early patterns of tactile behavior, for example, the nature and degree of touch between parents and their child, predict the child's later tendencies to seek or avoid touching people outside the family (Deethardt and Hines, 1984). Touch defined as positive for children's physical and psychological development has been described as patting, stroking, holding hands, tickling, hugging, kissing, stroking, and physically guiding the child (Stansbury et al., 2012). Positive touch during childhood is associated with lower levels of depression and higher relationship satisfaction during adolescence and early adulthood (Takeuchi et al., 2010).

Measuring and assessing touch avoidance is important not only for a deeper understanding of human touch but also for the implications touch has on healthcare practices. Touch in nursing provides a deeper connection with patients and can improve the nurse-patient relationship (Bensing et al., 2013; Deledda et al., 2013; Stein-Parbury, 2013). Moreover, there is evidence that social touch improves well-being and physical recovery (Nabi et al., 2013). There is, however, an association between length of service and comfort in using touch aimed at emotional containment; moreover, female and male nurses differ in performing this type of touch (Trifiletti et al., 2017). Even if most of the time social touch is appreciated by patients, an ignored area of investigation is why touch and massage sometimes fail in their soothing function. For instance, hand massage or foot massage in elderly care sometimes reduce agitation and improve well-being, but sometimes elicit opposite responses. These different responses seem to be associated with lack of experience or lack of confidence with the person who administered the massage, and with gender-mediated responses (Snyder et al., 1995; Moyle et al., 2014). Measuring attitudes toward touch could be important for helping young healthcare professionals to explore their automatic responses in using relational and caring touch, for better understanding when social and comforting touch is efficient and beneficial for patients, and when, in touch avoidant individuals, touch causes anxiety and aversion.

Assessing and measuring touch avoidance could also be helpful in psychotherapeutic settings (Perrella, 2017; Rohner et al., 2019). Clinicians often meet people struggling with romantic relationships, sexuality, or assertiveness. Often, clinicians' focus is on top-down processes: beliefs, motivations, and expectations that influence the patient's approach to other people and the patient's own responses. However, this approach may ignore how basal attitudes toward touch influence their patient's interpersonal relationships, social contexts, and bonding. Additionally, on the part of the patient, it can be useful to increase awareness and acceptance of their automatic responses to touch, experiencing their sensations and bodies in a non-judgmental way. This may help them inhibit automatic and dysfunctional responses to their unpleasant physical reactions, which could lead them to avoid meaningful relationships altogether. The role of touch in shaping and maintaining relationships has been explored in many studies of attachment. Touch seems strictly related to attachment style, a useful measure to predict how people experience intimate relationships during adulthood. Greenspan and Bowlby (1974) claimed that human 
touch facilitates the bond between a child and their caregiver and is essential for the child's well-being, especially in their early years. Anxious or avoidant individuals report a higher level of touch avoidance (Anderson, 1987; Brennan et al., 1998). Moreover, anxious individuals avoid intimacy, while dismissing individuals avoid closeness (Bartholomew and Shaver, 1998). Nelson and Geher (2007) showed that individuals who scored higher on anxiety subscales of an attachment questionnaire (worried style) reported more frequent cuddle-seeking behavior. Finally, individuals avoiding touch seem to need more intimacy in relationship, as a proof of their partner's love (Johansson, 2013).

To measure touch avoidance, Andersen and Leibowitz (1978) built a self-report instrument: the Touch Avoidance Measure (TAM), assessing attitudes toward and comfort with touch. The TAM is one of the most frequently used scales for measuring this trait. The scale analyzes two dimensions of touch avoidance: same-sex touch avoidance and opposite-sex touch avoidance. Another, more recent instrument is the Touch Avoidance Questionnaire (TAQ), developed by Ozolins and Sandberg (2009) a wide-ranging instrument that measures attitudes toward touch. The scale includes items that assess several social contexts of touch, including touch with a romantic partner, family (parents, siblings), same- and opposite-sex friends, and with strangers.

The aim of this study is to translate and validate in Italian these two questionnaires.

\section{MATERIALS AND METHODS}

\section{Procedure}

The study involved 335 participants (216 female, 113 male, six undisclosed; age $=35.82 \pm 14.32$; range, $16-74)$ recruited through convenience sampling drawing on the authors' personal networks. Participation was voluntary and anonymous, and participants received no compensation. The presentation order of the tests (all pencil-and-paper) was counterbalanced. Fortyone participants (30 female, eight male, three undisclosed; age, $27.84 \pm 9.30$; range, 20-55) were contacted a week after to do the test-retest, and 1 month after the first administration of the test to complete again the TAM and the TAQ.

Estimating an adequate sample size for a CFA depends on several aspects, including factor loadings, number of indicators per factor, estimator used, and the fit indices being considered (Kyriazos, 2018). Common, conservative rules of thumb for choosing adequate sample size for a CFA include ensuring that $N>300$ (Tabachnick and Fidell, 2012) and that the ratio $N / p$ (where $p$ is the number of indicator variables) is above 10 (Wang and Wang, 2012). Additionally, Tinsley and Tinsley (1987) suggested that an $N / p$ ratio between 5 and 10 can be adequate when $N>300$. In our case, the $N / p$ ratio is 18.6 for the TAM and 10.8 for the TAQ. All missing data (1.9\% for the TAM and $2.4 \%$ for the TAQ) were handled through pairwise deletion when estimating the correlation matrices.

Sample size was also adequate for the test-retest reliability estimates, following Hertzog (2008) recommendations.
The Ethics Committee of the University of Campania "Luigi Vanvitelli," Department of Psychology, approved this study. Recruiting and testing conformed with the local Ethics Committee requirements and the Declaration of Helsinki.

\section{Measures}

In 1978, Andersen and Leibowitz created the TAM to assess attitudes toward touch and comfort levels regarding touch. This instrument is composed of 18 statements concerning feelings about touching other people and being touched. Participants answer each item by rating it on a one- to five-point Likert scale (1 "fully disagree"; 5 "fully agree"). The measure is composed of two subscales: same sex (10 items, e.g., "Touching a friend of the same sex does not make me uncomfortable") and opposite sex (eight items, e.g., "When a member of the opposite sex touches me, I find it unpleasant").

In 2009, Ozolins and Sandberg developed the TAQ in order to assess level of touch avoidance in different contexts, such as situations involving romantic partner, siblings, parents, friends, professional touch, and touch with complete strangers. Some of the questions concerned attitudes toward touch with a samesex friend and some with a friend of the opposite sex. Across these categories, there were different questions about touching and receiving touch. The TAQ consists of 31 Likert-type items to which participants are asked to respond on a five-point scale (1 "fully disagree"; 5 "fully agree"). Items are divided in the subscales Partner (10 items, e.g., "I wish my partner would hold me for hours"), Family (six items, e.g., "I grew up in a cuddly family"), Same sex (six items, e.g., "I like to hug a same-sex friend"), opposite-sex (six items, e.g., "I try to avoid touch with an opposite-sex friend"), and Stranger [three items, e.g., "I find it very unpleasant to be in contact with unknown people (e.g., in queues, on the bus)"].

We translated the two scales from English to Italian; subsequently, an English native language speaker proceeded with the back translation; finally, we asked the authors of the TAM and the TAQ if the back translation was adequate. The Italian version of the questionnaires is included in Supplementary Material.

\section{Data Analysis}

Overall, questionnaire responses indicated a high degree of nonnormality for both the TAM and the TAQ (see Table 1) and the presence of considerable ceiling/floor effects. Mardia's tests for multivariate skew and kurtosis are significant for both the TAM $($ skew $=2738.44, p<0.001$, kurtosis $=21.04, p<0.001)$ and the TAQ (skew $=14826.86, p<0.001$, kurtosis $=30.56$, $p<0.001)$. For this reason, we opted to treat the items as ordinal, fitting confirmatory factorial analyses (CFAs) that would not be biased by the skewed distribution of data. However, a simple CFA with weighted least square mean and variance adjusted estimator would not converge due to: (1) the high number of parameters, since the model would require estimation of four threshold parameters for each item, and (2) the lack of observations for some response categories (i.e., 5 or 1) for some of the items due to ceiling/floor effects. Therefore, before fitting the CFAs, we recoded item responses as "low" (1 or 2 ), "medium," (3) or "high" (4 or 5). This would require the 
TABLE 1 | Descriptive statistics for Touch Avoidance Measure (TAM) and Touch Avoidance Questionnaire (TAQ) items.

\begin{tabular}{|c|c|c|c|c|c|c|c|c|c|c|c|}
\hline & Mean & SD & Median & Skew & Kurtosis & & Mean & SD & Median & Skew & Kurtosis \\
\hline$T A Q_{-1}$ & 3.51 & 1.09 & 4 & -0.52 & -0.58 & TAQ_31 & 3.86 & 0.85 & 4 & -1.26 & 2.39 \\
\hline TAQ_2 & 1.87 & 0.86 & 2 & 1.19 & 1.73 & TAQ_32 & 3.74 & 0.96 & 4 & -0.95 & 0.72 \\
\hline TAQ_3 & 1.45 & 0.89 & 1 & 2.35 & 5.28 & TAQ_33 & 1.94 & 1.00 & 2 & 1.08 & 0.71 \\
\hline TAQ_4 & 2.09 & 1.09 & 2 & 0.82 & -0.26 & TAQ_34 & 2.00 & 1.11 & 2 & 1.00 & 0.15 \\
\hline TAQ_5 & 1.63 & 0.89 & 1 & 1.61 & 2.40 & TAQ_35 & 2.49 & 1.09 & 2 & 0.38 & -0.54 \\
\hline TAQ_7 & 3.47 & 1.09 & 4 & -0.60 & -0.41 & TAQ_37 & 3.39 & 1.13 & 4 & -0.32 & -0.70 \\
\hline TAQ_8 & 3.99 & 0.81 & 4 & -0.66 & 0.36 & TAM_1 & 4.05 & 0.88 & 4 & -1.05 & 1.27 \\
\hline TAQ_9 & 1.39 & 0.63 & 1 & 1.81 & 4.21 & TAM_2 & 3.45 & 0.78 & 3 & -0.49 & 0.88 \\
\hline TAQ_11 & 1.86 & 1.02 & 2 & 0.99 & -0.15 & TAM_3 & 3.15 & 1.12 & 3 & -0.11 & -1.02 \\
\hline TAQ_14 & 2.03 & 1.05 & 2 & 0.82 & -0.06 & TAM_4 & 1.43 & 0.76 & 1 & 2.04 & 4.28 \\
\hline TAQ_16 & 3.38 & 1.28 & 4 & -0.36 & -1.00 & TAM_5 & 3.28 & 1.02 & 3 & -0.61 & -0.21 \\
\hline TAQ_17 & 3.10 & 1.29 & 3 & -0.04 & -1.14 & TAM_6 & 3.99 & 0.84 & 4 & -0.90 & 1.14 \\
\hline TAQ_18 & 3.65 & 1.16 & 4 & -0.73 & -0.27 & TAM_7 & 1.67 & 0.86 & 1 & 1.37 & 1.71 \\
\hline TAQ_19 & 2.78 & 1.26 & 3 & 0.14 & -1.08 & TAM_8 & 1.91 & 0.99 & 2 & 1.08 & 0.66 \\
\hline TAQ_20 & 2.74 & 1.35 & 3 & 0.14 & -1.34 & TAM_9 & 3.31 & 1.12 & 3 & -0.41 & -0.53 \\
\hline TAQ_21 & 2.20 & 1.28 & 2 & 0.81 & -0.55 & TAM_10 & 2.94 & 1.17 & 3 & -0.14 & -0.91 \\
\hline TAQ_22 & 1.82 & 1.00 & 2 & 1.34 & 1.27 & TAM_11 & 2.19 & 1.29 & 2 & 0.74 & -0.67 \\
\hline TAQ_23 & 3.62 & 1.07 & 4 & -0.83 & 0.16 & TAM_12 & 2.98 & 1.13 & 3 & -0.31 & -0.79 \\
\hline TAQ_24 & 1.93 & 1.03 & 2 & 1.10 & 0.60 & TAM_13 & 3.78 & 1.01 & 4 & -1.08 & 0.97 \\
\hline TAQ_25 & 3.94 & 0.86 & 4 & -1.22 & 2.46 & TAM_14 & 4.74 & 0.51 & 5 & -2.04 & 5.00 \\
\hline TAQ_26 & 3.71 & 0.98 & 4 & -0.89 & 0.63 & TAM_15 & 3.78 & 1.09 & 4 & -0.73 & -0.16 \\
\hline TAQ_27 & 1.94 & 1.01 & 2 & 1.16 & 0.97 & TAM_16 & 1.96 & 1.00 & 2 & 1.19 & 1.18 \\
\hline TAQ_28 & 2.22 & 1.10 & 2 & 0.68 & -0.48 & TAM_17 & 3.93 & 1.12 & 4 & -1.14 & 0.59 \\
\hline TAQ_29 & 3.65 & 0.97 & 4 & -0.78 & 0.26 & TAM_18 & 2.27 & 1.09 & 2 & 0.72 & -0.21 \\
\hline TAQ_30 & 2.03 & 1.05 & 2 & 0.91 & 0.04 & & & & & & \\
\hline
\end{tabular}

estimation of only two thresholds for each item, increasing the degrees of freedom and thus reducing the risk of fit indexes inflation due to overparameterization. Moreover, after recoding, all response categories had data, allowing CFA models to converge. For evaluating goodness of fit, we followed $\mathrm{Hu}$ and Bentler's (1999) conservative recommendations of comparative fit index (CFI) and Tucker-Lewis index (TLI) $>0.95$ and root-mean-square error of approximation (RMSEA) $<0.06$ as indicating excellent fit, guidelines widely followed in modern SEM research (Boduszek et al., 2016; Willmott et al., 2018).

Touch Avoidance Questionnaire items 6, 10, 12, 13, 15, and 36 were excluded from the final version of the test by the original authors (Ozolins and Sandberg, 2009).

After confirming the original models, the reliability of the scales was measured using ordinal alpha for each subscale of the tests (Gadermann et al., 2012) as well as test-retest reliability after 1 week and 1 month.

Subsequently, we tested convergent validity by computing the correlations between the Same Sex subscales of the two tests, and between their Opposite Sex subscales.

All data analyses were conducted using R (Version 3.6.2).

\section{RESULTS}

\section{Confirmatory Factor Analysis}

For both questionnaires, we tested the models conceptualized for their respective original versions, i.e., a two-factor model for the TAM (attitudes toward same sex touch and opposite sex touch) and a five-factor model for the TAQ (attitudes toward touch with one's partner, their same/opposite sex friends, their family, and strangers).

We observed good, but not excellent, fit for the TAM $[\mathrm{CFI}=0.947, \mathrm{TLI}=0.940, \mathrm{RMSEA}=0.065$, standardized rootmean-square residual $(\mathrm{SRMR})=0.138]$. All factor loadings were statistically significant, and their sign was the same as in the original scale. Correlation between the two factors was 0.56 . Examination of model parameters identified a relatively low loading for item 14 (0.176, see Table 2). The loading is significantly above 0 , but the estimate is below the oftenused cutoff of 0.300 . Additionally, examination of the highest modification indexes suggested adding correlations between the errors of items 7 and 8 and adding a loading for item 2 on the Same Sex factor. We did not apply these model modifications. Goodness-of-fit measures, even if slightly below $\mathrm{Hu}$ and Bentler's suggested cutoffs, still indicate relatively good fit. When considering the trade-off implicated in improving goodness of fit by editing the model, making comparisons with studies employing the English version of the TAM impossible, we deemed it better to err on the side of preserving the original model. This decision was also informed by the lack of theoretical grounding for adding the loading of item 2 on the Same Sex factor.

The confirmatory factor analysis for the TAQ, instead, achieved excellent fit, except for SRMR (CFI $=0.954, \mathrm{TLI}=0.950$, RMSEA $=0.058$, SRMR $=0.125)$. All factor loadings were 
TABLE 2 | Factor loadings for the Touch Avoidance Measure (TAM) and Touch Avoidance Questionnaire (TAQ).

\begin{tabular}{|c|c|c|c|c|c|c|c|}
\hline Item & Factor & Loading & $p$ & Item & Factor & Loading & $p$ \\
\hline TAQ_1 & TAQ Partner & 0.798 & $<0.001$ & TAQ_31 & TAQ Opposite Sex & 0.691 & $<0.001$ \\
\hline TAQ_2 & TAQ Partner & -0.649 & $<0.001$ & TAQ_32 & TAQ Opposite Sex & 0.904 & $<0.001$ \\
\hline TAQ_3 & TAQ Partner & -0.481 & $<0.001$ & TAQ_33 & TAQ Opposite Sex & -0.854 & $<0.001$ \\
\hline TAQ_4 & TAQ Partner & -0.610 & $<0.001$ & TAQ_34 & TAQ Stranger & 0.541 & $<0.001$ \\
\hline TAQ_5 & TAQ Partner & -0.796 & $<0.001$ & TAQ_35 & TAQ Stranger & 0.826 & $<0.001$ \\
\hline TAQ_7 & TAQ Partner & 0.851 & $<0.001$ & TAQ_37 & TAQ Stranger & 0.448 & $<0.001$ \\
\hline TAQ_8 & TAQ Partner & 0.548 & $<0.001$ & TAM_1 & TAM Same Sex & 0.409 & $<0.001$ \\
\hline TAQ_9 & TAQ Partner & -0.787 & $<0.001$ & TAM_2 & TAM Opposite Sex & 0.740 & $<0.001$ \\
\hline TAQ_11 & TAQ Partner & -0.601 & $<0.001$ & TAM_3 & TAM Same Sex & 0.509 & $<0.001$ \\
\hline TAQ_14 & TAQ Partner & -0.330 & $<0.001$ & TAM_4 & TAM Same Sex & -0.660 & $<0.001$ \\
\hline TAQ_16 & TAQ Family & 0.824 & $<0.001$ & TAM_5 & TAM Opposite Sex & 0.841 & $<0.001$ \\
\hline TAQ_17 & TAQ Family & 0.842 & $<0.001$ & TAM_6 & TAM Same Sex & 0.655 & $<0.001$ \\
\hline TAQ_18 & TAQ Family & 0.712 & $<0.001$ & TAM_7 & TAM Opposite Sex & -0.738 & $<0.001$ \\
\hline TAQ_19 & TAQ Family & 0.732 & $<0.001$ & TAM_8 & TAM Opposite Sex & -0.505 & $<0.001$ \\
\hline TAQ_20 & TAQ Family & -0.692 & $<0.001$ & TAM_9 & TAM Same Sex & 0.743 & $<0.001$ \\
\hline TAQ_21 & TAQ Family & -0.817 & $<0.001$ & TAM_10 & TAM Opposite Sex & 0.672 & $<0.001$ \\
\hline TAQ_22 & TAQ Same Sex & -0.748 & $<0.001$ & TAM_11 & TAM Same Sex & 0.607 & $<0.001$ \\
\hline TAQ_23 & TAQ Same Sex & 0.773 & $<0.001$ & TAM_12 & TAM Same Sex & 0.887 & $<0.001$ \\
\hline TAQ_24 & TAQ Same Sex & -0.799 & $<0.001$ & TAM_13 & TAM Same Sex & 0.666 & $<0.001$ \\
\hline TAQ_25 & TAQ Same Sex & 0.603 & $<0.001$ & TAM_14 & TAM Opposite Sex & 0.176 & 0.024 \\
\hline TAQ_26 & TAQ Same Sex & 0.871 & $<0.001$ & TAM_15 & TAM Opposite Sex & 0.716 & $<0.001$ \\
\hline TAQ_27 & TAQ Same Sex & -0.830 & $<0.001$ & TAM_16 & TAM Same Sex & -0.479 & $<0.001$ \\
\hline TAQ_28 & TAQ Opposite Sex & -0.731 & $<0.001$ & TAM_17 & TAM Opposite Sex & 0.542 & $<0.001$ \\
\hline TAQ_29 & TAQ Opposite Sex & 0.827 & $<0.001$ & TAM_18 & TAM Same Sex & -0.562 & $<0.001$ \\
\hline TAQ_30 & TAQ Opposite Sex & -0.923 & $<0.001$ & & & & \\
\hline
\end{tabular}

TABLE 3 | Correlations between Touch Avoidance Questionnaire (TAQ) factors.

\begin{tabular}{|c|c|c|c|c|c|}
\hline & Partner & Same Sex & Opposite Sex & Family & Stranger \\
\hline Partner & 1 & 0.29 & 0.13 & 0.26 & -0.30 \\
\hline Same Sex & 0.29 & 1 & 0.53 & 0.29 & -0.45 \\
\hline Opposite Sex & 0.13 & 0.53 & 1 & 0.13 & -0.61 \\
\hline Family & 0.26 & 0.29 & 0.13 & 1 & -0.40 \\
\hline Stranger & -0.30 & -0.45 & -0.61 & -0.40 & 1 \\
\hline
\end{tabular}

TABLE 4 | Test-retest correlation after 1 week and 1 month for all Touch Avoidance Measure (TAM) and Touch Avoidance Questionnaire (TAQ) subscales.

\begin{tabular}{lcc}
\hline Subscale & $\begin{array}{c}\text { 1 Week test-retest } \\
\text { Pearson's correlation }\end{array}$ & $\begin{array}{c}\text { 1 Month test-retest } \\
\text { Pearson's correlation }\end{array}$ \\
\hline TAM Same Sex & $0.91(0.84,0.96)$ & $0.87(0.75,0.93)$ \\
TAM Opposite Sex & $0.80(0.63,0.89)$ & $0.70(0.47,0.84)$ \\
TAQ Partner & $0.82(0.70,0.91)$ & $0.81(0.61,0.91)$ \\
TAQ Same Sex & $0.88(0.77,0.94)$ & $0.87(0.73,0.94)$ \\
TAQ Opposite Sex & $0.81(0.64,0.90)$ & $0.67(0.39,0.84)$ \\
TAQ Family & $0.94(0.88,0.97)$ & $0.90(0.79,0.95)$ \\
TAQ Stranger & $0.89(0.78,0.94)$ & $0.82(0.65,0.92)$ \\
\hline
\end{tabular}

statistically significant and above-in absolute value-the cutoff of 0.300 (see Table 2). The signs of all loadings were the same as in the original scale. Correlations between TAQ factors are reported in Table 3.
Models for the two questionnaires were fitted separately. TAQ items $6,10,12,13,15$, and 36 were excluded from the final version of the test by the original authors (Ozolins and Sandberg, 2009).

\section{Reliability}

Ordinal alpha was satisfactory for all but one of the subscales of the TAM and the TAQ. Specifically. for the TAM, we observe $\alpha=0.85(0.82,0.87)$ for the Same Sex subscale and $\alpha=0.84(0.81$, 0.87 ) for the Opposite Sex subscale (in the original validation paper, $\alpha=0.82$ and 0.88 , respectively). Ordinal $\alpha$ for the whole scale is $0.88(0.86,0.90)$. The high internal consistency for the whole scale is not only due to inflation of $\alpha$ for scales with more items (Kopalle and Lehmann, 1997) but also due to high correlation between the two TAM factors (0.559).

For the TAQ, we observe an ordinal $\alpha=0.84(0.81,0.87)$ for the Partner subscale, ordinal $\alpha=0.89(0.86,0.91)$ for the Same Sex subscale, ordinal $\alpha=0.92(0.90,0.93)$ for the Opposite Sex subscale, ordinal $\alpha=0.88(0.86,0.91)$ for the Family subscale, 

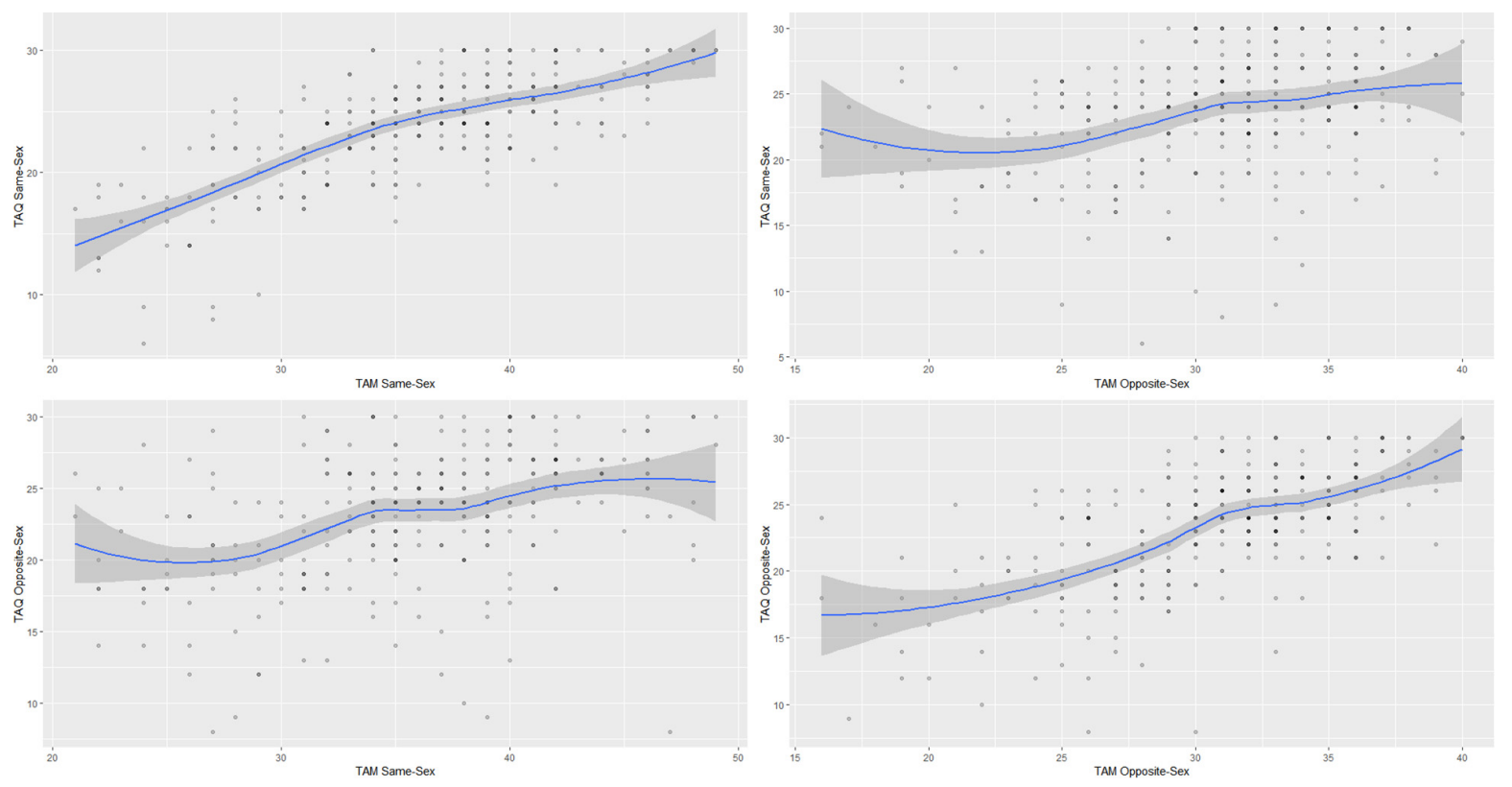

FIGURE 1 | Scatterplots for the Same Sex and Opposite Sex subscales of the Touch Avoidance Measure (TAM) and Touch Avoidance Questionnaire (TAQ). The polynomial regression curve was added to show the approximate linearity of relationship between subscales. The shaded area represents the $95 \%$ confidence area for the regression curve.

and ordinal $\alpha=0.59(0.54,0.70)$ for the Stranger subscale. The low value of $\alpha$ for the latter subscale may be because the subscale consists of only three items and $\alpha$ is sensitive to scale length. However, Spearman-Brown "prophecy" formula (Brown, 1910; Spearman, 1910) would predict an $\alpha$ as low as 0.74 were to subscale to comprise six items, suggesting that the Stranger subscale does have relatively low internal consistency. Ordinal $\alpha$ for the whole TAQ is $0.89(0.85,0.91)$. For comparison, in the original validation paper, $\alpha$ s were 0.86 (Partner), 0.89 (Same Sex), 0.85 (Opposite Sex), 0.85 (Family), and 0.64 (Stranger).

Test-retest reliability was examined by computing Pearson's correlation for the factor scores measured at each measurement time. Reliability was satisfactory for all subscales, ranging from 0.80 to 0.94 for 1 -week retest, and from 0.67 to 0.90 for 1 -month retest (see Table 4). All correlations are significant for $p<0.001$.

\section{Convergent Validity}

The correlation between the Same Sex subscales of the TAM and TAQ is $0.72(0.66,0.77), p<0.001$. The correlation between the Opposite Sex subscales for the two questionnaires is 0.63 $(0.56,0.70), p<0.001$. These results are in line with the TAQ validation study (Ozolins and Sandberg, 2009) which found correlations of 0.62 and 0.57 for the Same Sex and Opposite Sex scales, respectively. Inspection of item content reveals different conceptualization of the factors between the two tests: the TAQ always investigates attitudes toward being touched by friends of the same (or opposite) sex; the TAM, instead, usually refers to "people" of the same (opposite) sex, therefore investigating attitudes toward being touched by strangers.

The correlation between the Same Sex subscale of the TAM and the Opposite Sex subscale of the TAQ is $0.40(0.30,0.49)$, $p<0.001$, while the correlation between the Opposite Sex subscale of the TAM and the Same Sex subscale of the TAQ is $0.34(0.23,0.43), p<0.001$. These magnitudes seem to be consistent with those that would be expected for correlated constructs measured with different questionnaires adopting slightly different definitions. See Figure 1 for the scatterplots between these subscales.

\section{DISCUSSION}

In this study, we translated and validated two measures of touch avoidance-the TAM and the TAQ-for use on the Italian population. Overall, both validations can be considered successful: both the TAM and the TAQ models achieved good fit, 1-week and 1-month test-retest reliabilities are high, and convergent validity results are in the expected direction and magnitude. However, there are a few caveats that should be considered when using the TAM and TAQ with Italian samples.

First and foremost, goodness-of-fit indexes for the TAM were below $\mathrm{Hu}$ and Bentler (1999) cutoffs for excellent fit, and inspection of model parameters and modification indexes suggested to slightly modify the original model. We opted not to enact these modifications, as the model fit was still relatively good, with the rationale of providing Italian researchers with an instrument as close as possible to the original one for comparison and legacy purposes. However, we strongly suggest to use the TAQ, unless there are compelling reasons to use the TAM (e.g., for the replication of a study that used the TAM). This suggestion is borne not only of statistical considerations: TAM's domain is narrower than TAQ's, as it investigates touch avoidance only in the case of same 
and opposite sex people, without considering the additional settings investigated by the TAQ (strangers, family, partner). Additionally, some of TAM's items do appear a little dated, either in formulation or due to the changes in societal norms that occurred between 1978 and today.

Employing the TAQ therefore seems to be preferred, although it should be kept in mind that its "Stranger" subscale appears to have relatively low internal consistency and Opposite Sex subscale may have lower test-retest reliability than the others. Despite these minor concerns, the scale can be useful for investigating attitudes toward touch either in healthcare or in psychotherapeutic settings, so as to inform practitioners in the best course of action with their patients.

Subsequent studies could focus on investigating how touch avoidance varies in the Italian population according to gender, region of origin, age, or personality traits. Additionally, testing for measurement invariance for age and gender, as well as between the English and Italian versions of the tests could improve comparability of results across different populations.

\section{DATA AVAILABILITY STATEMENT}

The datasets presented in this study can be found in online repositories. The names of the repository/repositories and accession number(s) can be found below: https:/github.com/MPass/Touch-Avoidance-data.

\section{REFERENCES}

Andersen, P. A., and Leibowitz, K. (1978). The development and nature of the construct touch avoidance. Environ. Psychol. Nonverbal Behav. 3, 89-106. doi: 10.1007/BF01135607

Anderson, P. C. (1987). Touching: the human significance of the skin. JAMA J. Am. Med. Assoc. 257:2223. doi: 10.1001/jama.1987.03390160109042

Bartholomew, K., and Shaver, R. P. (1998). "Methods of assessing adult attachment," in Attachment Theory and Close Relationships, eds J. A. Simpson and S. W. Rholes (New York, NY: Guilford Press), 25-45.

Bensing, J., Rimondini, M., and Visser, A. (2013). What patients want. Patient Educ. Couns. 90, 287-290. doi: 10.1016/j.pec.2013.01.005

Boduszek, D., Debowska, A., Dhingra, K., and DeLisi, M. (2016). Introduction and validation of psychopathic personality traits scale (ppts) in a large prison sample. J. Crim. Justice 46, 9-17. doi: 10.1016/j.jcrimjus.2016.02.004

Brennan, K. A., Clark, C. L., and Shaver, P. R. (1998). "Self-report measurement of adult attachment: an integrative overview," in Attachment theory and close relationships, eds J. A. Simpson and S. W. Rholes (New York, NY: Guilford Press), 46-76.

Brown, W. (1910). Some experimental results in the correlation of mental abilities. Br. J. Psychol. 1920, 296-322. doi: 10.1111/j.2044-8295.1910.tb00207.x

Croy, I., Sehlstedt, I., Wasling, H. B., Ackerley, R., and Olausson, H. (2019). Gentle touch perception: from early childhood to adolescence. Dev. Cogn. Neurosci. 35 , 81-86. doi: 10.1016/j.dcn.2017.07.009

Deethardt, J. F., and Hines, D. G. (1984). Tactile communication and personality differences. J. Nonverbal Behav. 8, 143-156. doi: 10.1007/BF00987000

Deledda, G., Moretti, F., Rimondini, M., and Zimmermann, C. (2013). How patients want their doctor to communicate. A literature review on primary care patients' perspective. Patient Educ. Couns. 90, 297-306. doi: 10.1016/j.pec.2012. 05.005

Dibiase, R., and Gunnoe, J. (2004). Gender and culture differences in touching behavior. J. Soc. Psychol. 144, 49-62. doi: 10.3200/SOCP.144.1.49-62

\section{ETHICS STATEMENT}

The studies involving human participants were reviewed and approved by the Ethics Committee of the University of Campania "Luigi Vanvitelli," Department of Psychology. The patients/participants provided their written informed consent to participate in this study.

\section{AUTHOR CONTRIBUTIONS}

All authors conceived and designed the study, organized and supervised data collection and imputing, drafted the manuscript, organized and supervised the data analysis, and read and agreed to the published version of the manuscript.

\section{FUNDING}

GA was supported by the Ministry of Health under Grant Number GR-2018-12366092.

\section{SUPPLEMENTARY MATERIAL}

The Supplementary Material for this article can be found online at: https://www.frontiersin.org/articles/10.3389/fpsyg. 2020.01673/full\#supplementary-material

Ellingsen, D.-M., Leknes, S., Løseth, G., Wessberg, J., and Olausson, H. (2016). The neurobiology shaping affective touch: expectation, motivation, and meaning in the multisensory context. Front. Psychol. 6:1986. doi: 10.3389/fpsyg.2015.01986

Elvemo, N. A., Landrø, N. I., Borchgrevink, P. C., and Håberg, A. K. (2015). Reward responsiveness in patients with chronic pain. Eur. J. Pain 19, 1537-1543. doi: $10.1002 /$ ejp. 687

Finnegan, R. (2005). “Tactile communication," in The Book of Touch, ed. C. Clarence (New York, NY: Berg), 18-25.

Gadermann, A. M., Guhn, M., and Zumbo, B. D. (2012). Estimating ordinal reliability for likert-type and ordinal item response data: a conceptual, empirical, and practical guide. Pract. Assess. Res. Eval. 17, 1-12. doi: 10.7275/ n560-j767

Gazzola, V., Spezio, M. L., Etzel, J. A., Castelli, F., Adolphs, R., and Keysers, C. (2012). Primary somatosensory cortex discriminates affective significance in social touch. Proc. Natl. Acad. Sci. U.S.A. 109, E1657-E1666. doi: 10.1073/pnas. 1113211109

Greenspan, B., and Bowlby, J. (1974). Separation: anxiety and anger (Attachment and Loss-Volume II). Fam. Coord. 23:428. doi: 10.2307/583128

Hall, J. A., Coats, E. J., and LeBeau, L. S. (2005). Nonverbal behavior and the vertical dimension of social relations: a meta-analysis. Psychol. Bull. 131, 898-924. doi: 10.1037/0033-2909.131.6.898

Hertenstein, M. J., Holmes, R., McCullough, M., and Keltner, D. (2009). The communication of emotion via touch. Emotion 9, 566-573. doi: 10.1037/ a0016108

Hertenstein, M. J., Keltner, D., App, B., Bulleit, B. A., and Jaskolka, A. R. (2006). Touch communicates distinct emotions. Emotion 6, 528-533. doi: 10.1037/ 1528-3542.6.3.528

Hertzog, M. A. (2008). Considerations in determining sample size for pilot studies. Res. Nurs. Health 31, 180-191. doi: 10.1002/nur.20247

Hielscher, E., and Mahar, D. (2017). An exploration of the interaction between touch avoidance and the pleasant touch (C-Tactile Afferent) System. Perception 46, 18-30. doi: 10.1177/0301006616661938 
Hu, L., and Bentler, P. M. (1999). Cutoff criteria for fit indexes in covariance structure analysis: conventional criteria versus new alternatives. Struct. Equ. Model. A Multidiscip. J. 6, 1-55. doi: 10.1080/10705519909540118

Johansson, C. (2013). Views on and perceptions of experiences of touch avoidance: an exploratory study. Curr. Psychol. 32, 44-59. doi: 10.1007/s12144-0129162-1

Kopalle, P. K., and Lehmann, D. R. (1997). Alpha inflation? The impact of eliminating scale items on Cronbach's alpha. Organ. Behav. Hum. Decis. Process. 70, 189-197. doi: 10.1006/obhd.1997.2702

Kyriazos, T. A. (2018). Applied psychometrics: sample size and sample power considerations in factor analysis (EFA, CFA) and SEM in General. Psychology 09, 2207-2230. doi: 10.4236/psych.2018.98126

McDaniel, E., and Andersen, P. A. (1998). International patterns of interpersonal tactile communication: a field study. J. Nonverbal Behav. 22, 59-75. doi: 10. 1023/A:1022952509743

McGlone, F., Wessberg, J., and Olausson, H. (2014). Discriminative and affective touch: sensing and feeling. Neuron 82, 737-755. doi: 10.1016/j.neuron.2014.05. 001

Morrison, I., Löken, L. S., and Olausson, H. (2010). The skin as a social organ. Exp. Brain Res. 204, 305-314. doi: 10.1007/s00221-009-2007-y

Moyle, W., Cooke, M. L., Beattie, E., Shum, D. H. K., O’Dwyer, S. T., and Barrett, S. (2014). Foot massage versus quiet presence on agitation and mood in people with dementia: a randomised controlled trial. Int. J. Nurs. Stud. 51, 856-864. doi: 10.1016/j.ijnurstu.2013.10.019

Nabi, R. L., Prestin, A., and So, J. (2013). Facebook friends with (health) benefits? Exploring social network site use and perceptions of social support, stress, and well-being. Cyberpsychology, Behav. Soc. Netw. 16, 721-727. doi: 10.1089/cyber. 2012.0521

Nelson, H., and Geher, G. (2007). Mutual grooming in human dyadic relationships: an ethological perspective. Curr. Psychol. 26, 121-140. doi: 10.1007/s12144007-9009-3

Olausson, H., Wessberg, J., Morrison, I., McGlone, F., and Vallbo, A (2010). The neurophysiology of unmyelinated tactile afferents. Neurosci. Biobehav. Rev. 34, 185-191. doi: 10.1016/j.neubiorev.2008.09.011

Ozolins, A., and Sandberg, C. (2009). Development of a multifactor scale measuring the psychological dimensions of touch avoidance. Int. J. Psychol. A Biopsychosoc. Approach 3, 33-56.

Perrella, R. (2017). A Psychotherapy perspective: what about on the process and on the outcome in a functional approach for clinical and personality disorders? Mediterr. J. Clin. Psychol. 5, 1-11. doi: 10.6092/2282-1619/2017.5. 1662

Pizzagalli, D. A., Iosifescu, D., Hallett, L. A., Ratner, K. G., and Fava, M. (2008). Reduced hedonic capacity in major depressive disorder: evidence from a probabilistic reward task. J. Psychiatr. Res. 43, 76-87. doi: 10.1016/j.jpsychires. 2008.03.001

Rohner, R. P., Filus, A., Melendez-Rhodes, T., Kuyumcu, B., Machado, F., Roszak, J., et al. (2019). Psychological maladjustment mediates the relation between remembrances of parental rejection in childhood and adults' fear of intimacy: a multicultural study. Cross Cult. Res. 53, 508-542. doi: 10.1177/ 1069397118822992

Scheele, D., Kendrick, K. M., Khouri, C., Kretzer, E., Schläpfer, T. E., StoffelWagner, B., et al. (2014). An oxytocin-induced facilitation of neural and emotional responses to social touch correlates inversely with autism traits. Neuropsychopharmacology 39, 2078-2085. doi: 10.1038/npp.2014.78
Sehlstedt, I., Ignell, H., Backlund Wasling, H., Ackerley, R., Olausson, H., and Croy, I. (2016). Gentle touch perception across the lifespan. Psychol. Aging 31, 176-184. doi: 10.1037/pag0000074

Serino, A., and Haggard, P. (2010). Touch and the body. Neurosci. Biobehav. Rev. 34, 224-236. doi: 10.1016/j.neubiorev.2009.04.004

Snyder, M., Egan, E. C., and Burns, K. R. (1995). Efficacy of hand massage in decreasing agitation behaviors associated with care activities in persons with dementia. Geriatr. Nurs. 16, 60-63. doi: 10.1016/S0197-4572(05)80005-9

Spearman, C. (1910). Correlation calculated from faulty data. Br. J. Psychol. 3, 271-295. doi: 10.1111/j.2044-8295.1910.tb00206.x

Stansbury, K., Haley, D., Lee, J., and Brophy-Herb, H. E. (2012). Adult caregivers' behavioral responses to child noncompliance in public settings: gender differences and the role of positive and negative touch. Behav. Soc. Issues 21, 80-114. doi: 10.5210/bsi.v21i0.2979

Stein-Parbury, J. (2013). Patient and Person: Interpersonal Skills in Nursing. Chatswood, AU: Elsevier.

Tabachnick, B. G., and Fidell, L. S. (2012). Using multivariate statistics, 6th Edn. Manhattan, NY: Harper \& Row.

Takeuchi, M. S., Miyaoka, H., Tomoda, A., Suzuki, M., Liu, Q., and Kitamura, T. (2010). The effect of interpersonal touch during childhood on adult attachment and depression: a neglected area of family and developmental psychology? J. Child Fam. Stud. 19, 109-117. doi: 10.1007/s10826-009-9290-x

Thomsen, K. R. (2015). Measuring anhedonia: impaired ability to pursue, experience, and learn about reward. Front. Psychol. 6:1409. doi: 10.3389/fpsyg. 2015.01409

Tinsley, H. E., and Tinsley, D. J. (1987). Uses of factor analysis in counseling psychology research. J. Couns. Psychol. 34, 414-424. doi: 10.1037/0022-0167. 34.4.414

Trifiletti, E., Pedrazza, M., Berlanda, S., and Pyszczynski, T. (2017). Burnout disrupts anxiety buffer functioning among nurses: a three-way interaction model. Front. Psychol. 8:1362. doi: 10.3389/fpsyg.2017.01362

Vallbo, ÅB., Olausson, H., and Wessberg, J. (1999). Unmyelinated afferents constitute a second system coding tactile stimuli of the human hairy skin. J. Neurophysiol. 81, 2753-2763. doi: 10.1152/jn.1999.81.6.2753

Wang, J., and Wang, X. (2012). Structural Equation Modeling. Chichester: : John Wiley \& Sons, Ltd.

Wilhelm, F. H., Kochar, A. S., Roth, W. T., and Gross, J. J. (2001). Social anxiety and response to touch: incongruence between self-evaluative and physiological reactions. Biol. Psychol. 58, 181-202. doi: 10.1016/S0301-0511(01)00113-2

Willmott, D., Boduszek, D., Debowska, A., and Woodfield, R. (2018). Introduction and validation of the juror decision scale (JDS): an empirical investigation of the Story Model. J. Crim. Justice 57, 26-34. doi: 10.1016/j.jcrimjus.2018.03.004

Conflict of Interest: The authors declare that the research was conducted in the absence of any commercial or financial relationships that could be construed as a potential conflict of interest.

Copyright (C) 2020 Casetta, Rizzi, Passarelli, Arcara and Perrella. This is an openaccess article distributed under the terms of the Creative Commons Attribution License (CC BY). The use, distribution or reproduction in other forums is permitted, provided the original author(s) and the copyright owner(s) are credited and that the original publication in this journal is cited, in accordance with accepted academic practice. No use, distribution or reproduction is permitted which does not comply with these terms. 